\title{
Infections in children with asthma
}

\author{
Abdullah Abdulrahman Al Shimemeri \\ Department of Intensive Care Medicine, Intensive Care Department, College of Medicine, King Saud bin Abdulaziz University for Health \\ Sciences, Riyadh, Kingdom of Saudi Arabia
}

\section{Email address:}

aftercom@yahoo.com

\section{To cite this article:}

Abdullah Abdulrahman Al Shimemeri. Infections in Children with Asthma. International Journal of Immunology.

Vol. 2, No. 1, 2014, pp. 1-5. doi: 10.11648/j.iji.20140201.11

\begin{abstract}
Asthma is one of the most common chronic disorders in childhood. Asthma symptoms normally find in first three years of a life when the growth and remodeling of lungs is at maximum pace. There is always a common concern regarding different forms of asthma and how to cure them. Studies showed us in US about 20 million people are suffering from asthma and out of which 9 millions are children. Globally around 70\% of asthmatic children are suffering from Wheeze which is mostly a symptom of viral infection. This is also one of the global burdens due to respiratory viral infections imposed by asthma. The various types of infections include rhinovirus infections which plays a crucial role in asthma development; respiratory syncytial virus which cause lower respiratory tract infection such as bronchiolitis; influenza and par- influenza viruses; pertussis, one of the major reason of death for children below 3 months of age; epiglottitis; bronchiolitis which effects mainly children below age of 2; and pneumonia caused by bacteria, fungi, parasites or due to virus. In this article we will shed some light on the relationship of chest infections with asthma and how they impact the course of asthma disease in children. The most important factor is how several vitamins like Vitamin D plays a crucial role for asthma patients. General studies show prevention and long term control is major key in stopping asthma attacks. There are various medications available to avoid asthma attacks but it generally depends upon the patient's symptoms, age and the various triggering factors. Further we will see how a balanced diet helps like pro-biotic supplements, bacterial derived product, OM-85 etc helps in controlling asthma infections.
\end{abstract}

Keywords: Asthma, Children, Bronchiolitis, Syncytial Virus, Pneumonia

\section{Introduction}

Asthma is a protracted disease that causes airway narrowing and inflammation. It is characterized by periods of recurring wheezing, chest tightening, coughing and breathes shortness. Persistent coughing is more prolonged at night and early in the morning. ${ }^{1}$

It is reported that in the United States alone, about 20 million of the population suffer from asthma with close to 9 million being children due to smaller airways compared to adults. ${ }^{2}$ Many young children of today have different types of exposures to environmental and infections as those children who lived in the past years. The exposure adversely affects the immune systems development during very early childhood and increase atopy and asthma risks in them. This is the case with children who have genetic background of asthmatic conditions. ${ }^{3}$ Young children with respiratory infections and wheeze are more at the highest risks of contracting asthma that persist beyond the age of 6 . Allergies, eczema or parents with asthma are the unfavorable conditions that will encourage asthma inception ${ }^{4}$

Lung function testing is the best asthma diagnostic which involves investigating medical history and performing an in-depth physical screening. Asthmatic symptoms one has, the frequency and severity of will differ from one patient to another. Sometimes, the symptoms will irritate the subject while in other times may hinder your normal lifestyle and could even be fatal if they are extremely severe. ${ }^{5}$

The best measures to control both short and long term effects of asthma are to prevent it from even starting. Treatment will only be through understanding and avoiding agents that triggers asthma, learning how to breathe to keep asthma conditions at bay. When attacked by asthma some inhalers come handy such as albuterol. Medication is dependent on several factors such as age, asthmatic triggers, symptoms and your effective asthmatic reliever controls. If administered for a long time, medication will reduce respiratory inflammation such as long-acting beta agonists, leukotriene modifiers, inhalers combination and theophylline. For bronchodilators it includes short-acting beta agonists, 
ipratropium, oral and intravenous corticosteroids. Medications for allergy can help if your asthma is allergy triggered and involves using allergy shots commonly called immunotherapy, omalizumab and other allergy medications. In the case of severe asthma bronchial, thermoplasty is used because it require more than inhaled corticosteroids or even medications meant for long-term asthma . ${ }^{6}$

Asthmatic triggers are categorized into two: allergic and non-allergic They results in inflammation and tightening of muscles in the airway. These triggers are virtually everywhere such as dust mites or even activities that are not allergic such as physical exercise, infections by viruses and other irritants. Any prevention strategy should focus on identifying the non-allergic and allergic triggers and avoiding them. Between $80-90 \%$ of asthmatic adult patients have allergic asthma triggered by allergens that include pollen dusts, dander and dusts mites. ${ }^{7}$

\section{Role of Infections in Asthma}

Asthma exacerbation is caused mainly by viral infections to respiratory organs and increases the risks of children at younger age who have genetic susceptibility to catching asthma. In the case of acute asthma exacerbations, they are associated with lungs which are not fully grown or those which have lost much of their functions and are therefore a contributing factor to the cost and prevalence due to asthma. Although drastic measures to combat viral infections have been formulated, those strategies have not been utilized effectively. Observing high standards of personal hygiene, washing of hands and keeping away from cigar smoke are a few of the measurers that if taken will significantly minimize viral infections in the respiratory tissues. Observation of a balanced healthy with active supplements of probiotic and products that are bacteria-free will minimize the chances of infection recurrence in vulnerable children. The human immune system that is responsible for body defense against viral infection has both specific and non-specific components. In children who are asthmatic, they can improve their immune functionality by having an healthy lifestyle coupled with physical exercise on a regular basis, eating a balanced diet, getting enough sleep and keeping off causative agents such staying in an environment filled with smoke from tobacco, stress or taking avoidable antibiotics.

The first 2-3 years are important because it is during this time that asthma occurrence is high as lungs starts to develop and airways are remodeled at a fastest rate. Inflammation of pulmonary due to sLRI and atopy during this susceptibility time is believed to confuse differentiation of underlying tissues causing destructive effects which is long term on the functions of respiratory. ${ }^{8}$

Viral infections to the respiratory tract are believed to be mechanisms behind occurrence of asthma and viruses have been identified to be associated with asthma in at least three instances: a given virus has been shown to be behind asthmatic phenotype that causes genetic vulnerability to asthmatic symptoms and attack. The other reason is the increased chances of repeated infections due to exposure from sources around the subject although it can work to reduce risk of asthma and allergy complications due to completely new change in response profile of cytokines or pre-existing ones. Finally, patients with asthmatic history, especially in children, tract infections to the upper respiratory are responsible for acute exacerbation due to obstruction of airway because of interaction with hospital environment or visits from outpatients. In this review we will dwell on data that is available about viral infections to the respiratory syncytial and association to inception of asthma predominantly during childhood. ${ }^{9}$

\section{Rhinovirus Infections and the Development of Asthma}

It has been found out that rhinovirus infections are responsible in a way for development of asthma, asthmatic exacerbations and remodeling of airway. Children who suffer from wheezing bouts during infancy that is induced by rhinovirus will be at a greater risk of asthma attack compared to those children who wheeze due to syncytial viral infection of respiratory tract. ${ }^{10} \mathrm{RV}$ infections will trigger many changes of immune system in the airways or blood and some of the responses correlates with symptoms of cold, airways responsiveness changes or symptoms of lower-airways. ${ }^{11}$

\section{Respiratory syncytial virus (RSV)}

This is a well-known cause of infections to the LRTIs (lower respiratory tracts). The respiratory syncytial virus (RSV) is greatly associated with lower respiratory tract infection that includes bronchiolitis in young children and infants. Statistically, close to $70 \%$ of infants get RSV infection during the first year their life and by the age 2 years, almost all children have been infected at least once. Mechanisms through which RSV infection could raise vulnerability to asthma have been proposed and include chronic epithelial and reactivity changes in airway to the still developing lung of an infant, lung injury that alters functioning of lung and changes to immunomodulatory. ${ }^{12}$

\section{Influenza and Parainfluenza Viruses}

Acute infections by influenza causing viruses can amplify inflammation of immune airway in many of the patients with asthma. This disease exacerbate the normal symptoms exhibited in respiratory infections and responsiveness of bronchial to stimuli allergy. ${ }^{13}$ Respiratory tract infections, influenza in particular causes about $80 \%$ of acute exacerbations of asthma. Asthmatic patients are more susceptible to developing influenza with severe problems of the disease. Incidence of viral respiratory infections of the tract is similar to asthmatic subject is similar as that public at large but chances of contracting lower respiratory tract 
illness is twice higher due to reduced viral clearance in the airway. Patients with asthma that have exacerbations reoccurrence will suffer persistent damage to epithelium of the airway that will lead to minimized function of airway as airway clearance is very poor. ${ }^{14}$

\section{Pertussis (Whooping Cough)}

Pertussis is a bacterial disease that affects the respiratory cavities and is infectious. The name whooping cough is coined from the term "whoop" characterized by spasms of coughing which is punctuated by a sound when child deeply inhales after a prolonged coughing spell. Whooping cough prevalence in infants and children is on the rise. Infants who are under the age 3 months suffer most deaths from pertussis.

15 Asthmatic patients have 1.7 times higher prevalence compared to those without, pointing to the fact that asthma escalates the risk of one suffering from whooping cough. ${ }^{16}$ Child vaccinations is the most preferred prevention measures to curb incidences of whopping cough occurrence in children and it is recommended between the ages of two, three, and four months as one of the 'five-valent' vaccinations against pertussis, polio, diphtheria, tetanus, and Hib vaccine.

\section{Epiglottitis}

This is a life-threatening problem that is characterized by the swelling of the epiglottis and in the process blocking air flow into lungs. In the past in children were infected by Haemophilus influenzae type b (Hib), a bacterium that causes meningitis, pneumonia, and bloodstream infections. 17 When left untreated, epiglottitis will cause a number of complications that includes respiratory failure and in severe cases spreads multiple infections in the entire body. Documented studies and reports by FDA reveal that on 14th of August 2013: $13(0.03 \%)$ of the 49,599 people with asthma were suffering from epiglottitis. The relationship between asthma and epiglottitis is unclear and needs further researching.

\section{Bronchiolitis}

This is the swelling and buildup of mucus in the tight air valves in the lungs, mostly because of an infection by virus. It affects children below the age of 2 ; those between 3-6months are at most risk. It normally occurs but may get severe. Sometimes and the RSV (Respiratory syncytial virus) is the most common contributor. Currently no RSV vaccine is available but there is an alternative product that has proven effective called palivizumab that is used in high risk infants susceptible to more acute disease caused by RSV. ${ }^{18}$ Bronchiolitis and asthma share symptoms that are similar. Although there is no evidence to show that bronchiolitis directly causes asthma, or if even children who later suffer from asthma were likely to get bronchiolitis at infancy, asthma is severe and subjects suffer from it longer than bronchiolitis. Children suffering from bronchiolitis are at higher risk to contract asthma at one time in life. ${ }^{19}$

\section{Pneumonia}

Pneumonia is lungs infection caused by micro-organisms such viruses, bacteria, fungi, or parasites. It manifests mainly through alveoli inflammation in the lungs or fluid filling the alveoli-microscopic sacs that absorbs oxygen from inhaled air to the lungs. People with pneumonia starts with flu and cold-like symptoms which advance to extreme fever, chills and cough accompanied by sputum.. ${ }^{20}$ Asthmatic individuals are at high risk to develop pneumonia compared to those who have no asthma. Also, increase in asthma attack to patients with asthma can be traced to pneumonia. Patients with who suffer from pneumonia attack while having asthma will give abnormal results when pulmonary-function tests is administered for months or even years after recovering from pneumonia. ${ }^{21}$ A FDA warning that some drugs administered to asthmatic patients may leave them more vulnerable to pneumonia infections. Pneumonia occurrence was twice the normal in subjects on LABA combination than patients on LABA alone when clinical studies were conducted using COPD inhaled steroids

\section{Relationship of Virus-Induced Wheezing in Early Life to Childhood Asthma}

It is common for viral respiratory infections that are associated with wheezing during infancy to reoccur later in young children which is the cause of hospitalizations. Although the problem will disappear in many children, a greater number of asthmatic children and those school going kids with under developed lung functions start wheezing during most of their infancy years. It remains an issue to debate whether the infections by symptomatic virus to the lower tracts of respiratory are intermittent in development of asthma or just identifies susceptible children. ${ }^{22}$

\section{Innate Immunity to Virus Infection}

The host innate immune systems, in the case of infections by virus, will be the first defense mechanism used to stop invasion by virus or their replication before the body uses more specific means for protection using the immune system that is adaptive in nature which is generated. Here, Pattern Recognition Receptors (PRSs) are tasked upon to identify presence of components such as viral DNA, RNA or products that are intermediate and viral specific. Type I interferons (IFNs) are induced and other cytokines which are pro-inflammatory in the immune cells or those infected. These immune receptors which are innate and unique with their downstream pathways have recently been found. ${ }^{23}$ These are important for early detection of viral invasion and immediate trigger of adaptive immune system. The three receptor classes are very essential in type IIFNs and 
pro-inflammation cytokines production. Plasmacytoid dendritic cells on the other hand make use of TLRs to detect invasion of viruses. NLRs are essential for mature interleukin-1 b production to stimulation of dsRNA activation of immune cells which are innate and very critical in triggering and subsequent release of immune responses that are adaptive

\section{Vitamin D}

Regardless of the significance of healthy bones, vitamin D is responsible for optimal function in adaptive and innate immune systems that includes dendritic cells development and antimicrobial proteins production by regulatory $\mathrm{T}$ lymphocytes that modifies inflammatory disorders of intestinal flora and inflammatory inflammation modulation response to infections by virus. Its insufficiency is associated with increased occurrence of infections to respiratory tracts, wheezing condition in children, control in asthma reductions, visits to emergency departments, asthma exacerbations severity and hospitalization. ${ }^{25}$

\section{Conclusion}

Every year during winter, parents with children suffering with asthma are concerned due to the risk of acute asthma exacerbations after a common cold. They are actually right because statistic reveal that $70 \%$ of children with asthma will exhibit intermittent symptoms after a respiratory infection by a virus. ${ }^{8}$ Respiratory viral infections contribute majorly to the burden caused by asthma around the globe. In infants, respiratory viral infections contribute to start of asthma and accounts for most of acute asthma exacerbations occurrence in children. ${ }^{8}$ The epidemiologic indications that strongly link virus with exacerbations has been observed and now the task is to identify important dynamics of asthma exacerbations that is caused by virus. Viral infections is suspected to cause inflammation of airways but no evidence have been given to distinguish between asthmatic patients from non-asthmatic individuals. ${ }^{26}$ Currently, no practical therapies is used against mass infections but research on more anti-viral therapies that are specific and even vaccinations are ongoing. Much academic interest has been given to the role of primary strategies employed in asthma prevention in susceptible children and results are expected from many studies underway. ${ }^{8}$

It has been shown also that lack of antiviral activity and the susceptibility of epithelial barrier along the airway could increase the risks of asthmatic individuals suffering severe viral infections of the respiratory and hence high risk of exacerbation. Efforts to know the factors causing changes in the pathophysiology of airway are the right path to take towards finding better treatment for asthma in view of the effects due to viral presence in the respiratory. ${ }^{27}$

\section{Abbreviations}

(RSV) Respiratory syncytial virus,

(LRTIs) lower respiratory tract infections,

(Hib) Haemophilus influenzae type b,

(PRRs) pattern recognition receptors,

(RNA) Ribonucleic acid,

(DNA) Deoxyribonucleic acid,

(IFNs) interferons,

(RIG-I) retinoic acid-inducible gene I,

(RLRs) retinoic acid-inducible gene I-like receptors,

(TLRs) Toll-like receptors,

(NOD) nucleotide oligomerization domain,

(NLRs) nucleotide oligomerization domain -like receptors,

(dsRNA) double-stranded RNA,

(dsRNA) double-stranded RNA.

\section{References}

[1] What is asthma? National Heart, Lung, and Blood Institute. http://www.nhlbi.nih.gov/health/health-topics/topics/asthma/. Accessed 26 August 2013

[2] Asthma in children. Medline Plus. http://www.nlm.nih.gov/medlineplus/asthmainchildren.html. Accessed 27 August 2013.

[3] What causes asthma? National Heart, Lung and Blood Institute.

http://www.nhlbi.nih.gov/health/health-topics/topics/asthma/ causes.html. Accessed 26 August 2013

[4] Who is at risk for asthma? National Heart, Lung and Blood Institute.

http://www.nhlbi.nih.gov/health/health-topics/topics/asthma/ atrisk.html. Accessed 26 August 2013

[5] What are the signs and symptoms of asthma? National Heart, Lung and Blood Institute. http://www.nhlbi.nih.gov/health/health-topics/topics/asthma/ signs.html. Accessed 27 August 2013.

[6] Treatments and drugs. Mayo Clinic staff. http://www.mayoclinic.com/health/asthma/DS00021/DSEC TION=treatments-and-drugs. Accessed 26 August 26, 2013.

[7] Asthma in infants and young children. Asthma Society of Canada.

http://www.asthma.ca/adults/control/pdf/Asthma_in_infants and_young_children_tip_sheet.pdf. Accessed $27^{-}$August 2013.

[8] Respiratory viral infections in children with asthma: do they matter and can we prevent them. BMC Pediatrics. http://www.biomedcentral.com/1471-2431/12/147. Accessed 27 August 2013. 
[9] Robert F. Lemanske Jr., Viral infections and asthma inception The journal of allergy and clinical immunology. http://www.jacionline.org/article/S0091-6749\%2804\%2902 272-9/abstract. Accessed 27 August 2013.

[10] Role of rhinovirus infection in asthma. PubMed.gov. http://www.ncbi.nlm.nih.gov/pubmed/22053589. Accessed 26 August 2013

[11] James E. Gern, William W. Busse. Association of rhinovirus infections with asthma. National Center for Biotechnology Information.

http://www.ncbi.nlm.nih.gov/pmc/articles/PMC88904/.

Accessed 26 August 2013.

[12] Pingsheng Wu. Tina V Hartert. Evidence for a causal relationship between respiratory syncytial virus infection and asthma. Expert Rev Anti Infect Ther. 2011 September; 9(9): 731-745.

[13] Girolamo Pelaia, Alessandro Vatrella, Luca Gallelli, Teresa Renda, Mario Cazzola, Rosario Maselli, Serafino A. Marsico. Respiratory infections and asthma. Respiratory Medicine Volume 100, Issue 5, May 2006, Pages 775-784

[14] Robbie D. Pesek, Richard F. Lockey. Influenza and asthma: a review. World Allergy Organization. http://www.worldallergy.org/professional/allergic_diseases_ center/influenza asthma/. Accessed 27 August $20 \overline{1} 3$.

[15] John P. Cunha. Whopping Cough (Pertussis). Medicine net. http://www.onhealth.com/whooping_cough_health/article.ht m. Accessed 26 August 2013.

[16] Asthma increases risk of whooping cough. American Academy of Allergy Asthma \& Immunology. http://www.aaaai.org/global/latest-research-summaries/Curr ent-JACI-Research/asthma-increase-whooping-cough.aspx. Accessed 26 August 2013.

[17] Epiglottitis. Mayo Clinic. http://www.mayoclinic.com/health/epiglottitis/DS00529. Accessed 27 August 2013.
[18] Bronchiolitis. U.S. National Library of Medicine. http://www.nlm.nih.gov/medlineplus/ency/article/000975.ht m. Accessed 27 August 2013.

[19] Bronchiolitis.

Kids

Health. http://kidshealth.org/parent/infections/lung/bronchiolitis.htm 1. Accessed 27 August 2013.

[20] What is pneumonia? What causes pneumonia? Medical News Today.

http://www.medicalnewstoday.com/articles/151632.php. Accessed 27 August 2013.

[21] What is the connection between pneumonia and asthma? Wise geek. http://www.wisegeek.com/what-is-the-connection-betweenpneumonia-and-asthma.htm. Accessed 27 august 2013.

[22] Jackson DJ, Lemanske RF Jr. The role of respiratory virus infections in childhood asthma inception. Pub Med. http://www.ncbi.nlm.nih.gov/pubmed/21029935. Accessed 27 August.

[23] Koyama S, Ishii KJ, Coban C, Akira S. Innate immune response to viral infections. Pub Med. http://www.ncbi.nlm.nih.gov/pubmed/18694646. Accessed 27 August 2013.

[24] Osamu Takeuchi, Shizou Akira. Innate immunity to virus infection.

http://cc.bjmu.edu.cn/download/1469d0793292486fb79de54 20688-2906309031657.pdf. Accessed 27 August 2013

[25] Respiratory viral infections in children with asthma: do they matter and can we prevent them. BMC Pediatrics. http://www.biomedcentral.com/1471-2431/12/147. Accessed 27 August 2013.

[26] Patrick Mallia, MD; Sebastian L. Johnston, MD, PhD How viral infections cause exacerbation of airway diseases. Chest. 2006; 130(4):1203-1210.

[27] William W Busse MD a, Robert F Lemanske MD a b, James E Gern MD. Role of viral respiratory infections in asthma and asthma exacerbations. Lancet, Volume 376 (2010), Issue 9743, Pages $826-834$ 